\title{
Viability of simultaneous analysis through reagent mixture in microfluidic paper-based analytical devices using colorimetric measures
}

\section{Wendel Ferreira Santos and Adriana Vitorino Rossi}

\begin{abstract}
In this project, the viability and adequacy of simultaneous analysis conducted in a single reactional zone of Microfluidic Paper-based Analytical Device ( $\mu \mathrm{PAD}$ ) though the mixure of 2 or more colorimetric reagents was evaluated for synthetic samples with the concentration range of 0 to $100 \mathrm{ppm}$ for the analytes. The analytical system used in this research was based on a combination of the aluminum chloride method to quantify flavonoids through complex formation and the Folin-Ciocalteu reagent method to quantify the total phenolic content through redox reactions. Both methods were adapted from their original spectrophotometric systems to new microfluidic ones.
\end{abstract}

\section{Key words: \\ microfluidic, simultaneous analysis, $\mu P A D$}

\section{Introduction}

Antioxidants are compounds responsible for the inhibition or delay of oxidation processes promoted by free radicals in other molecules. Considering a living organism, the metabolic and respiratory activities generate radical species of oxygen (ROS) that may harm biomolecules present inside cells if the quantity generated is not minimized. Thus, the accumulation of these species may trigger a series of diseases like diabetes mellitus, Parkinson, Alzheimer, and even cancer. ${ }^{1}$ For these reasons, daily ingestion of antioxidant compounds is indispensable. These compounds may be divided between their origins: synthetic (phenolic compounds with varying degrees of alkyl substitution) and natural (like phenolic acids, flavonoids, carotenoids, and amino acids). Since legal restrictions on synthetic antioxidants have been established, natural sources have gained the spotlight and quantification methods have been develop for them. Along with the ever-increasing search for these new sources, portable devices, like Microfluidic Paperbased Analytical Devices ( $\mu \mathrm{PAD}$ ), that allow quantification would come in hand to evaluate if native species would be suitable to be used or not as a commercial raw material. In this project, the use of a mixture of 2 or more colorimetric reagents in a single reactional zone of a MPAD was tested to quantify simultaneously flavonoids and the total phenolic content in synthetic samples, using digital images scanning and the RGB color system.

\section{Results and Discussion}

The methods used to quantify flavonoids and total phenolic content in the microfluidic systems were adapted from spectrophotometric systems.

For the flavonoid determination, the aluminum chloride method was chosen. ${ }^{2}$ The quantification involves the formation of a yellow flavonoid-Al ${ }^{3+}$ complex whose color gets more intense the higher the concentration of the analyte (Image 1).

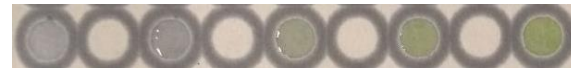

Image 1. Obtained colors in $\mu$ PAD for different concentration values of Quercetin through aluminum chloride method (from left to right: $0,25,50,75,100 \mathrm{ppm}$ )

For the total phenolic content determination, the Folin-Ciocalteu (FC) reagent method was chosen. ${ }^{3}$ The quantification involves a redox reaction that generates a colored product whose color gets more intense the higher the concentration of the analyte (Image 2).

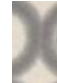

Image 2. Obtained colors for different concentration values of Gallic Acid through FC reagent method (from left to right: 0, 25, $50,75,100 \mathrm{ppm})$

For the simultaneous analysis of both analytes in a single reactional zone, some adaptations were needed due to compatibility issues between the systems. In order to avoid the precipitation of $\mathrm{Al}_{2} \mathrm{CO}_{3}$ and the lack of reactivity of the Folin-Denis reagent in low $\mathrm{pH}$, the buffer solutions (sodium carbonate and potassium acetate) were removed. The color scale obtained for some concentration combinations is shown in Image 3.

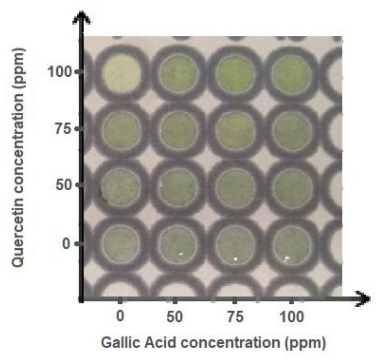

Image 3. Color scale obtained from the developed simultaneous analysis method with each axis containing different concentration values for gallic acid (horizontal) and quercetin (vertical)

\section{Conclusions}

Using the RGB color system, the correlations between the concentration and its most adequate color components for quantification were found, being them 0,970 (blue component) for flavonoids and 0,951 (green component) for total phenolic content. Both these values were obtained studying a concentration range of 0 to $100 \mathrm{ppm}$.

\section{Acknowledgement}

The sponsoring support from the CNPq is acknowledged.

\footnotetext{
${ }^{1}$ Gupta, R.K.; Patel, A.K.; Shah N.; Chaudhary, A.K.; Jha, U.K.; Yadav, U.C.; Gupta, P.K.; Pakuwal, U.; Asian Pac J Cancer Prev. 2014

2 Chang, C.C.; Yang, M.H.; Wen H.M.; Chern, J.C.; J. Food Drug Anal. 10 2002, 178-182.

3 Velioglu, Y.S.; Mazza, G.; Gao, L.; Oomah, B.D.; J. Agric. Food Chem. 46, 1998, 4113-4117.
} 